\title{
MAC Sub-Layer Analysis with Channel Estimation in Broadband Power Line Communication
}

\author{
Mohammad Khaled Andari ${ }^{1}$, Seyed Ali Asghar Beheshti ${ }^{2}$ \\ ${ }^{1}$ IEEE Member, Electrical Engineering Department, Iran University of Science and Technology, Tehran, Iran \\ ${ }^{2}$ Faculty Member, Electrical Engineering Department, Iran University of Science and Technology, Tehran, Iran \\ E-mail:m.kh.andari@ieee.org,abeheshti@iust.ac.ir \\ Received May 31, 2011; revised June 27, 2011; accepted July 4, 2011
}

\begin{abstract}
Broadband power line communication (BPLC) gained a lot of interest because of low cost and high performance communication network in access area. In this paper physical (PHY) layer and medium access control (MAC) sub-layer of BPLC are considered. Furthermore, effects of bit error rate (BER) are analyzed in MAC sub-layer. Powerful turbo convolutional code (TCC) and wideband orthogonal frequency division multiplexing (OFDM) are used in PHY layer. Carrier sense multiple access (CSMA) and virtual slot multiple access (VSMA) are taken into consideration in MAC sub-layer. Multilayered perceptrons neural network with backpropagation (BP) learning channel estimator algorithm compare to classic algorithm in for channel estimating. The simulation results show that the proposed neural network estimation decreases bit error rate then in MAC sub-layer throughput increases and access delay is decreased.
\end{abstract}

Keywords: BPLC, Back-Propagation Neural Network (BPNN), Channel Estimation, Throughput, Access Delay

\section{Introduction}

Nowadays high-speed communication on low-voltage power lines is getting more attention. Technological progress of access networks to bring broadband services is increasing very fast recently. Reasonably priced broadband Internet communication to residential customers is now available via cable modems and various flavors of Digital Subscriber Line (DSL). In turn there is a growing need for in-home network to share this single full-time Internet access link while supporting the wide range of digital data and multimedia communication services. Useful application of Power line bandwidth requires robust physical (PHY) and medium access control (MAC) protocols to mitigate the harsh and rough conditions of Power line channel. Orthogonal Frequency Multiple Access presents a good performance in BPLC channel which is time and frequency variant. In physical layer, HomePlug AV (HPAV) has 200 Mbps channel rate to provide a 150 Mbps information rate. Orthogonal frequency division multiplexing (OFDM) that is wide spread technology for many wireless standards, is used in HPAV standard that employs advanced PHY and MAC technologies in BPLC networks.
Turbo coding can be thought of as a refinement of concatenated encoding structure plus an iterative algorithm for decoding the associated code sequence. This code achieves a bit-error probability of $10^{-5}$ using a rate $1 / 2$ code over an additive Gaussian noise (AWGN) channel and BPSK modulation at an $\mathrm{E}_{\mathrm{b}} / \mathrm{N}_{0}$ of $0.7 \mathrm{~dB}$.

Channel impulse response (CIR) for BPLC channel can be estimated using predetermined pilot symbols in real time in exchange for information rate reduction. There are two types of techniques for channel estimation. One of them is blind channel estimation and other is pilot based channel estimation that is our desire in this paper. Blind channel estimation techniques attempt to guess the channel impulse responses only with transmitted data. Due to time varying of BPLC channel this channel estimation is not suitable. Channel estimation with pilot is the approach in which predetermined data are transmitted at the beginning of a frame data. This method has better performance than blind estimation in varying time and frequency channels [1]. In this paper we analysis three types of pilot base channel estimation, least square, minimum mean square error, and neural network.

Our proposed channel estimation is neural network with back-propagation learning method. 
LS is so easy for implementation and has no good performance. MMSE implementation needs noise and correlation computation, but it has respectable performance in time and frequency varying channel. Third method performance is between LS and MMSE (near to MMSE). The advantage of our method is we do not need knowledge of noise and other statistics of the channel. BPLC channel has various noises. These noises may be different in one channel to others. Because of this reason, having no knowledge of channel parameter our method is the best among these three methods. Disadvantage of neural network is it has more computation for training neural network. In contrast with wireless communication power line communication doesn't have power supply problem. However, more complexity is not a severe problem because it may be removed by powerful processors. Turbo code performance is compared with block code and uncoded data in BPLC physical layer.

In MAC sub-layer, VSMA algorithm which is used in IEEE 802.15.3 is taken into consideration. This algorithm is hybridized CSMA/CA and TDMA to improve throughput and access delay. In CSMA/CA, a binary exponential backoff algorithm and dynamic contention window is being employed. Furthermore influence of bit error rate in PHY and MAC layer is discussed. As a result of neural network channel estimation, the BER is decreased and after that throughput and access delay are improved.

The reminder of this paper organized as follow: in Section 2, systems physical layer and in Section 4 systems MAC sub-layer of BPLC channel is described. Channel estimation methods are considered in Section 3. Simulation result presented in Section 5, and finally Section 6 contains conclusions. Bold letters are chosen for notation of matrix and vector variables.

\section{Physical Layer}

The physical layer works in frequency range of $2 \mathrm{MHz}$ $28 \mathrm{MHz}$ and provides a $200 \mathrm{Mbps}$ channel rate and 150 Mbps information rate. It uses scrambler, wideband OFDM and robust turbo convolutional coding. This coding has performance near $0.5 \mathrm{~dB}$ less than Shannon capacity. One purpose of scrambling is to reduce the length of strings of $0 \mathrm{~s}$ or $1 \mathrm{~s}$ in a transmitted signal. Long string of $0 \mathrm{~s}$ or $1 \mathrm{~s}$ may cause transmission synchronization problems. OFDM provides flexible spectrum notching capability where the notches can exceed $30 \mathrm{~dB}$ in depth without losing significant useful spectrum outside the notch. Usually BPSK or QAM (like 1024 QAM) modulation are used for each subcarrier in OFDM. Random interleaving is done during turbo encoding to reduce effects of burst error. The Data mapper block accepts inte- ger inputs and produces integer outputs. We can select one of four mapping modes: Binary to Gray, Gray to Binary. For example, with binary to gray mapping vector $[0,1,2,3,4,5,6,7]$ is mapped to $[0,13,2,6,7,5,4]$.

Cyclic prefix is often used in conjunction with modulation in order to retain sinusoids' properties in multipath channels. The cyclic prefix serves two purposes. First, as a guard interval it eliminates the inter-symbol interference from the previous symbol. Second, as a repetition of the end of the symbol, it allows the linear convolution of a frequency-selective multipath channel to be modeled as circular convolution, which in turn may be transformed to the frequency domain using a discrete Fourier transform. This approach allows for simple frequency-domain processing, such as channel estimation and equalization. In order for the cyclic prefix to be effective (i.e. to serve its aforementioned objectives), the length of the cyclic prefix must be at least equal to the length of the multipath channel.

Peak limiter used for limiting OFDM signal's peak. In general, large signal peaks occur in the transmitted signal when the signals in many of the various sub-channels add constructively in phase. Such large signal peaks may result in clipping of the signal voltage in a D/A converter when the multicarrier signal is synthesized digitally, and/or it may saturate the power amplifier and thus cause inter-modulation distortion in the transmitted signal.

Analog front end (AFE) is the analog portion of a circuit which precedes A/D conversion. In receiver all of operations described above are done inversely. AVG block is automatic gain control. Figure 1 shows transmitter and receiver used for physical layer.

\subsection{Channel Model}

Indoor power line channel has frequency and time variant behavior. The power line link may be subjected to various constraints such as attenuation because of interference, multipath over the electrical wiring, or electrical

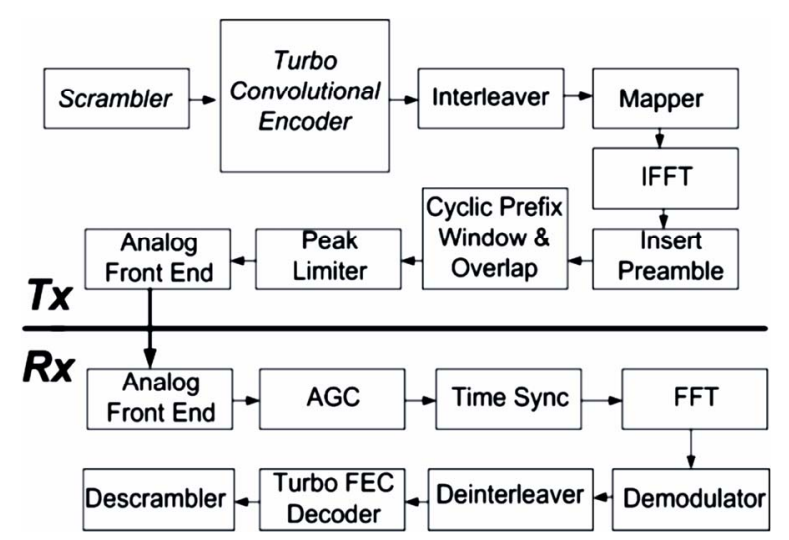

Figure 1. Physical layer transmitter and receiver. 
wiring crosstalk effects. Then, we can describe this channel like this (1).

$$
h(\tau ; t)=\sum_{p=0}^{N_{p}-1} \alpha_{p}(t) \delta\left(\tau-\tau_{p}(t)\right)
$$

where $\alpha_{p}$ is $p^{\text {th }}$ fading channel coefficient, $\mathrm{p}$ is path number, $N_{p}$ is number of multipath, $\tau_{p}$ is $p^{\text {th }}$ delay path. Fading channel coefficients are independent and is a Rician distributed random variable. Path delays are independent Gaussian random variables [2].

\subsection{Noise Model}

Additive white Gaussian noise is not the dominant noise in power line channels. The different types of noise that can be identified on and around the wiring are:

- Impulse noise from stops and starts of electrical devices.

- Broadband white noise whose power spectral density is the same for all frequencies (background noise).

- Periodic noise at multiple frequencies.

- Harmonic noise composed of multiple frequencies used by electrical equipment connected to the network and which are multiples of the line frequency (for example, $50 \mathrm{~Hz}$ yields harmonics of $300 \mathrm{~Hz}, 600$ $\mathrm{Hz}$, and so forth).

Overall, the noise is quantified by the signal-to-noise ratio, or SNR, generally measured in decibels $(\mathrm{dB})$ [8].

Model of the background noise is Nakagami-m that its PDF can be written as

$$
p(r)=\frac{2}{r(m)}\left(\frac{m}{\Omega}\right)^{m} r^{2 m-1} \mathrm{e}^{-\frac{m r^{2}}{\Omega}}
$$

where $r$ is a random variable, $r(\cdot)$ is the Gamma function, $p$ is the probability of the corresponding random variable, $m$ is defined as the ratio of moments, and $\Omega$ is the mean power of the random variable [9].

\section{Channel Estimation}

We derive three estimator based on physical layer and system model in previous section. Assume $X$ is Fourier transforming of the transmitted data. Then $Y$, the received vector in frequency domain, is: [7]

$$
y=X h+n
$$

or

$$
\left[\begin{array}{c}
y^{(1)} \\
\vdots \\
y^{(k)}
\end{array}\right]=\left[\begin{array}{lll}
x^{(1)} & & \\
& \ddots & \\
& & x^{(k)}
\end{array}\right]\left[\begin{array}{c}
h^{(1)} \\
\vdots \\
h^{(k)}
\end{array}\right]+\left[\begin{array}{c}
n^{(1)} \\
\vdots \\
n^{(k)}
\end{array}\right]
$$

where $h$ is frequency response of BPLC channel and $n$ is Fourier transform of additive BPLC channel noise.
OFDM modulation is used to transmit data. $k$ is number of sub-channels in the OFDM block. Since BPL channel is often a multipath fading channel, results to intersymbol interference (ISI) in the received signal. To remove ISI, various types of equalizers can be used before detection. Detection algorithms which based on trellis search (such as MLSE or MAP) have a good performance, but even now have too much computation. These detectors need information on channel impulse response (CIR) that can be provided by a separate channel estimator. Figure 2 shows block diagram for a system utilizing channel estimator [4].

\subsection{LS Estimation}

Consider the linear model [7]

$$
y=X h+m
$$

For given estimate of $h$, the squared error between $y$ and the model $X h$ is defined to be

$$
\begin{aligned}
e^{2} & =\operatorname{tr}\left[(y-X h)(y-X h)^{T}\right] \\
& =(y-X h)^{T}(y-X h)=n^{T} n
\end{aligned}
$$

This is optimized to obtain the least squares estimate. The appropriate equation for the gradient and second gradient of $e^{2}$ with respect to $h$ are

$$
\begin{gathered}
\frac{\partial}{\partial h} e^{2}=2 X^{T}(y-X h) \\
\frac{\partial}{\partial h}\left(\frac{\partial}{\partial h} e^{2}\right)^{T}=2 X^{T} X \geq 0
\end{gathered}
$$

The least squares estimate equates gradient to zero to produce the solution

$$
\hat{h}=\left(X^{T} X\right)^{-1} X^{T} y
$$

This solution exists and is unique, provided that the inverse of $X^{T} X$ exists.

We say that the least squares solution for $\hat{h}$ obeys

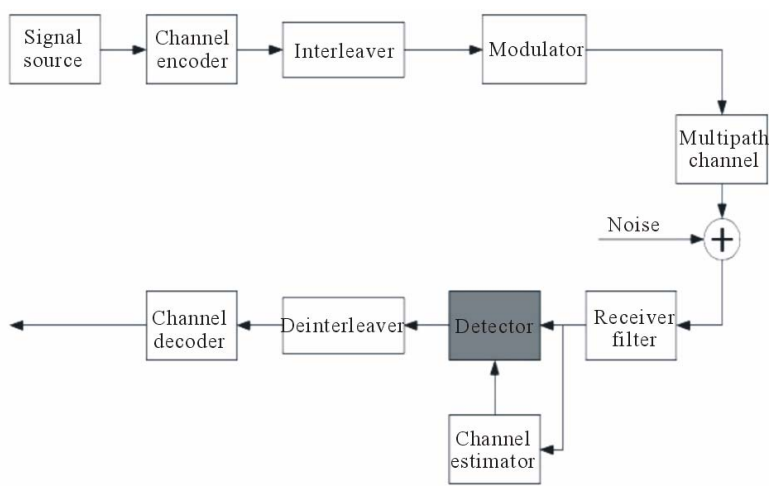

Figure 2. Block diagram for channel estimator. 
the normal equations

$$
X^{T} X \hat{h}=X^{T} y
$$

where the matrix $G=X^{T} X$ is called the Grammian or Gram matrix and the matrix $\left(X^{T} X\right)^{-1} X \quad 0$ is pseudo inverse of matrix $X$. If the Gram matrix is non-singular the solution for $\hat{h}$ is unique.

In this part $X$, is rectangular matrix but without lost of generality, in this paper we suppose that $X$ is square matrix. Then, we can rewrite Equation (9) like this

$$
\hat{h}_{l s}=X^{-1} y
$$

or like this

$$
\hat{h}_{l s}=\left[\begin{array}{llll}
\frac{y_{0}}{x_{0}} & \frac{y_{1}}{x_{1}} & \cdots & \frac{y_{N-1}}{x_{N-1}}
\end{array}\right]
$$

\subsection{MMSE Estimation}

In our channel model described, MMSE estimator is LMMSE estimator because of linearity. Thus error $h-\hat{h}$ is orthogonal to our received data $y$ so we can write [7]

$$
E\left\{(h-\hat{h}) y^{H}\right\}=0
$$

$\hat{h}$ is linear estimator of $y$ then

$$
\begin{aligned}
& E\left\{(h-k y) y^{H}\right\}=0 \\
& R_{h y}-K R_{y y}=0 \\
& K=R_{h y} R_{y y}^{-1}
\end{aligned}
$$

now we obtain

$$
\hat{h}_{m m s e}=R_{h y} R_{y y}^{-1} y
$$

where

$$
\begin{aligned}
R_{h y} & =E\left\{h y^{H}\right\}=R_{h h} x^{H} \\
R_{y y} & =E\left\{y y^{H}\right\}=X R_{h h} x^{H}+R_{n n} \\
& =X R_{h h} x^{H}+\sigma^{2} I
\end{aligned}
$$

$I$ is identity matrix and $R_{h h}$ is autocovariance matrix of channel coefficient. So we obtain Equation (17)

$$
\hat{h}_{\text {mmse }}=R_{h h} x^{H}\left(X R_{h h} x^{H}+\sigma^{2} I\right)^{-1} y
$$

Let us rewrite Equation (17) like this

$$
\hat{h}_{m m s e}=R_{h h} x^{H}\left(X R_{h h} x^{H}+\sigma^{2} I\right)^{-1} X X^{-1} y
$$

Suppose $A=x^{H}\left(X R_{h h} x^{H}+\sigma^{2} I\right)^{-1} X$. If we use this technique $A=\left(A^{-1}\right)^{-1}$, we can rewrite Equation (18) like this

$$
\hat{h}_{m m s e}=R_{h h}\left(R_{h h}+\sigma^{2}\left(X X^{-1}\right)^{H}\right)^{-1} \hat{h}_{l s}
$$

where

$$
\hat{h}_{l s}=X^{-1} y
$$

and

$$
\hat{h}_{m m s e}=R_{h h}\left(R_{h h}+\frac{\beta}{S N R} I\right)^{-1} \hat{h}_{l s}
$$

where

$$
\begin{aligned}
& E\left\{\left(X X^{H}\right)^{-1}\right\}=E\left\{\left|1 / x_{k}\right|^{2}\right\} I \\
& \beta=E\left\{\left|x_{k}\right|^{2}\right\} E\left\{\left|1 / x_{k}\right|^{2}\right\} \\
& S N R=E\left\{\left|x_{k}\right|^{2}\right\} / \sigma_{n}^{2}
\end{aligned}
$$

\subsection{BPNN Estimation}

To gain CIR, MLP neural network with backpropagation learning algorithm are used. Because of neural network nature, OFDM symbol which is a complex signal must be separated into real and imaginary parts. Neural network have two inputs and two outputs. Two outputs of neural network are real and imaginary part of CIR in the frequency domain. MLP structure for neural network channel estimation is shown in Figure 3 [6].

\section{MAC Sub-Layer}

MAC sub-layer provides both connection oriented and connectionless communication between source and destination station. For connection-oriented one uses time division multiple access (TDMA) and for other uses carrier sense multiple access collision avoidance (CSMA/ CA). VSMA algorithm is hybridized CSMA/CA with TDMA to guarantee latency and jitter requirement for real-time communication. In CSMA/CA for quality of service for priority is provided. The stations with higher priority can transmit their frame with more probability. Exponential backoff algorithm is done among station

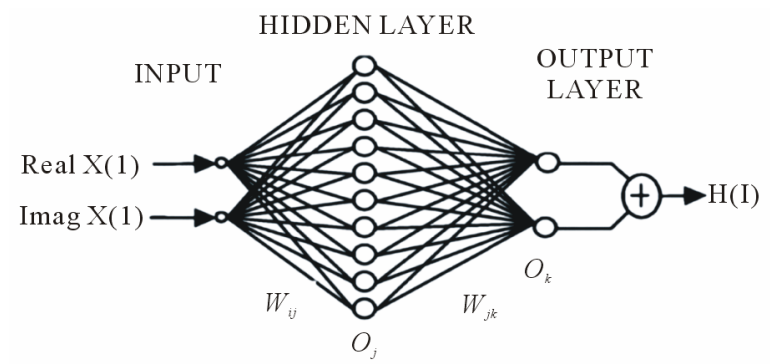

Figure 3. MLP structure for channel estimation. 
with the same priority. According to backoff algorithm, each station waits a random time after priority resolution (PR) then transmits its frame. Contention window (CW) size for a station is maximum time that station waits to transmit its frame. In Figure 4 timing of data transmission is shown. In this figure CIFS and RISF stand for contention inter frame spacing and response inter frame spacing, respectively. In [10] and [11] fixed and dynamic CW for VSMA algorithm is discussed. One can find details about VSMA in these two references. In this paper, influence of bit error rate in PHY layer is discussed. If BER in PHY layer is low then when a station access the medium can transmit frame without more error. With coded data, low error occurred in frame can be corrected and acknowledgement frame in RIFS can transmit to transmitter with high probability.

\section{Simulation Results}

In this part, numerical results are collected. Three channel estimations (LS, MMSE, and NN) are compared. Mean square error between channel coefficient $h$ and estimated channel coefficient $\hat{h}$ is gained with simulation. Figure 5 shows that performance of MMSE is better than LS and neural network (NN) estimation, and NN performance is better than LS estimation. In simulation, for all of estimators, channel estimations are done in frequency domain. To compare MSE simulation result with theoretical value, Cramer-Rao lower bound (CRLB) is plotted. Background noise is additive Gaussian noise. Without loss of generality, for simplification of simulation, we can suppose in only impulsive noise and AWGN noise are existed in power line. This is because of the fact that the other noise can be removed by powerful coding such as turbo coding. Impulsive noise can be made by another AWGN noise which is added to above noise. The power of this noise is 4 to 5 times greater than background noise with duration of about 2milliseconds. The modulation is BPSK and FFT size is 64 points. This means that in Equation (3) $k=64$. MLP network has one hidden layer with 10 neurons. In hidden layer sigmiod activation function and in output layer linear activation function is used. Radial basis function neural network performance with the same structure of BPNN is less than BPNN. We have used Monte-Carlo simulation to generate $R_{h h}$ for MMSE estimation. For drawing Figure 5 , simulation is repeated fifteen times and mean of the results are calculated. Figure 6 compares the performances of different modulation (BPSK, 4 QAM, QPSK and DPSK) without channel estimation in BPL physical layer. This comparison is done because of OFDM structure. In each sub-channel of OFDM, different modulation can be applied due to the condition of it. Figure 7 shows channel estimation comparison in presence of BPSK modulation.

In Figure 8, the performance of turbo coding with convolutional coding (with same generator $g=\left[\begin{array}{llll}1 & 1 & 1 ; 1 & 0\end{array}\right.$ $1])$ and $\mathrm{BCH}(15,7)$ is compared. Furthermore, coding

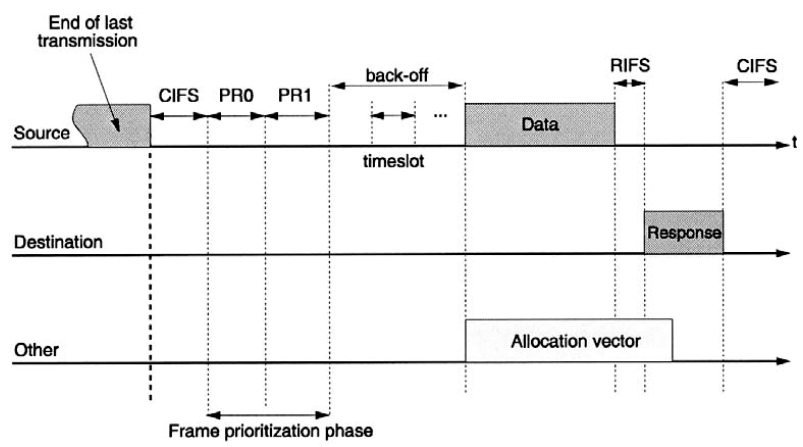

Figure 4. Timing of data transmission for a transmitter and a receiver.

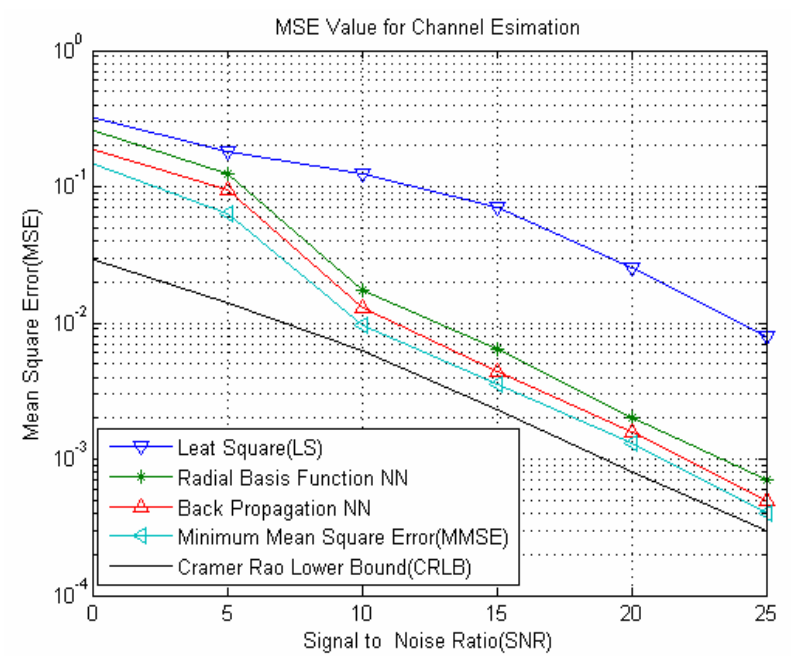

Figure 5. MSE values for channel estimation.

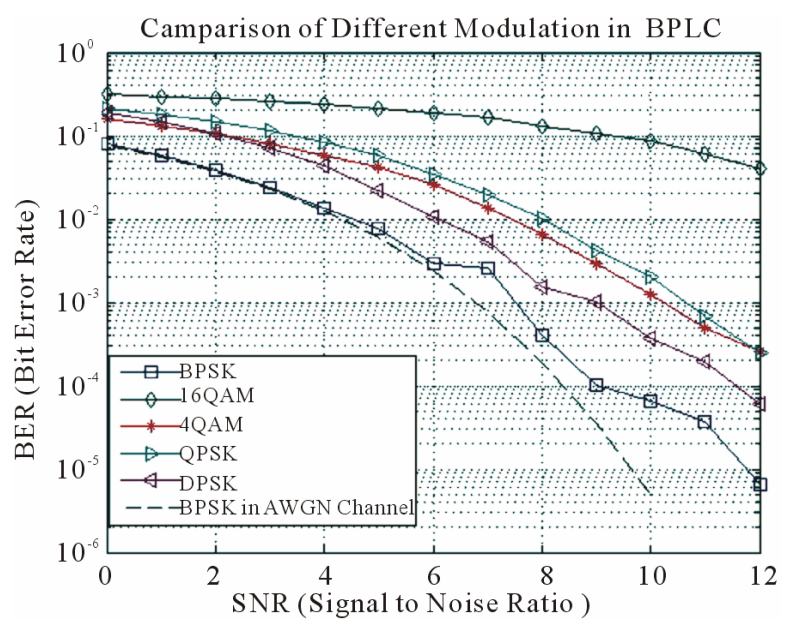

Figure 6. Different modulation in BPL physical layer. 
rate for all coding is $r=1 / 2$. In this part of simulation less than $2 \times 10^{8}$ binary data are transmitted. Some parameters that are needed for simulation of MAC layer are listed in Table 1.

Throughput vs. frame size and number of station for CSMA/CA at SNR $=10 \mathrm{~dB}$ are presented in Figure $\mathbf{9}$. Access delay time vs. number of station at the same SNR is available in Figure 10. For more analysis of MAC sub-layer, analysis of VSMA is done. Throughput of VSMA algorithm when BPNN channel estimation is done is much better than no channel estimation. Figure 12 shows throughput of VSMA without BPNN channel estimation and Figure 13 shows excess throughput of VSMA with BPNN channel estimation. In Figure 11, access delay before and after BPNN channel estimation are clearly shown. In order to analyze of VSMA at SNR $=10 \mathrm{~dB}$, as shown in Figure 7, BER $=3.923 \times 10^{-6}$ be fore channel estimation and BER $=7 \times 10^{-7}$ after BPNN channel estimation are taken.

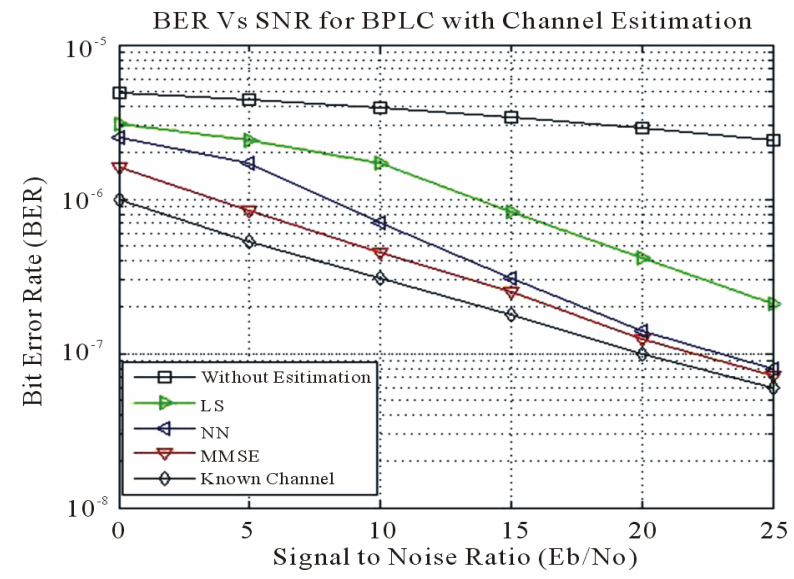

Figure 7. BER vs. SNR (dB) in different channel estimation in BPSK modulation.

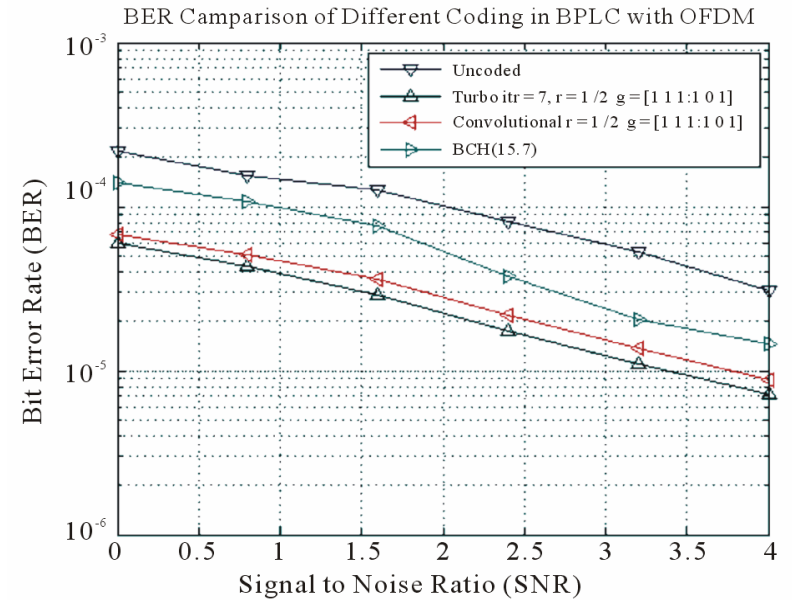

Figure 8. Different coding comparison in PHY layer.

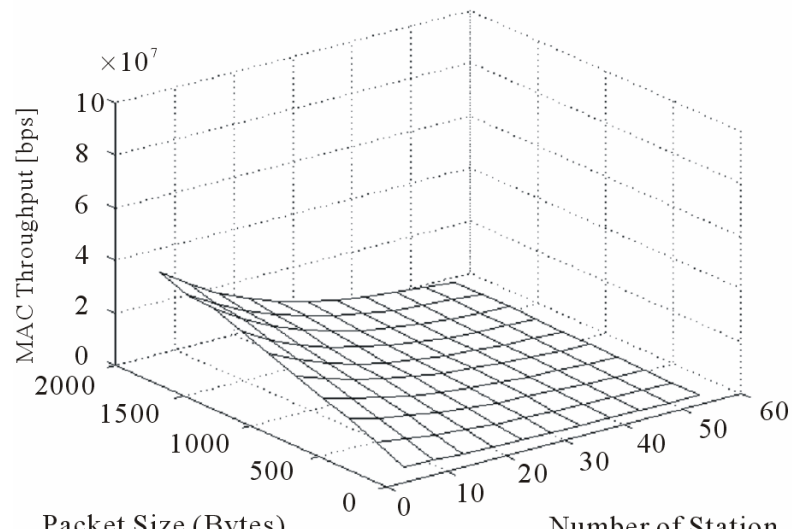

Figure 9. Throughput in CSMA/CA.

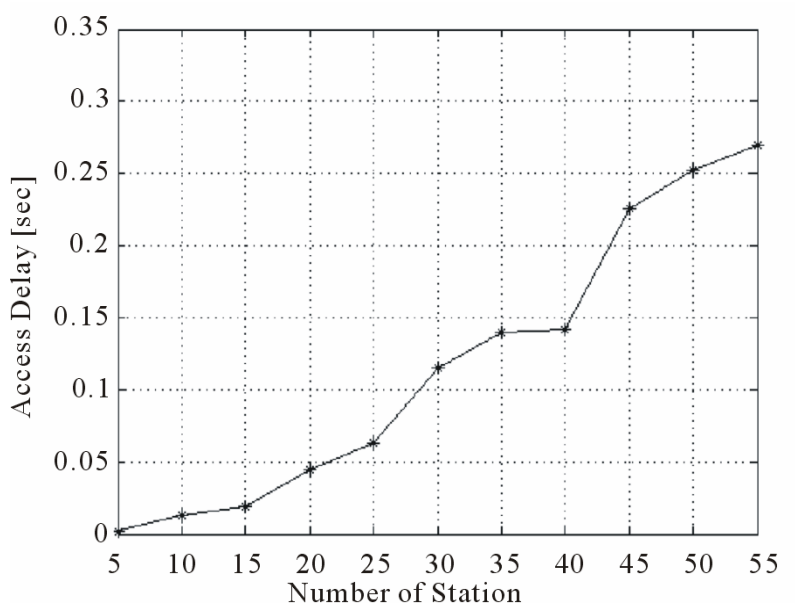

Figure 10. Access delay in CSMA/CA.

Table 1. Time slot values and other parameters used for simulation.

\begin{tabular}{cc}
\hline Protocol & VSMA \\
\hline Physical Channel Rate & $200 \mathrm{Mbps}$ \\
CIFS & $38.84 \mu \mathrm{sec}$ \\
PRS0, PRS1 & $38.84 \mu \mathrm{sec}$ \\
Idle Slot Time & $38.84 \mu \mathrm{sec}$ \\
RIFS & $38.84 \mu \mathrm{sec}$ \\
Mac Frame Size & $100-2000$ Byte \\
\hline
\end{tabular}

\section{Conclusions}

Citation powerline communication is becoming more and more popular in last mile that include home networking. MMSE estimator suffers from a high complexity; whereas the LS estimator has a high mean square error. This paper presents BPNNs channel estimation 


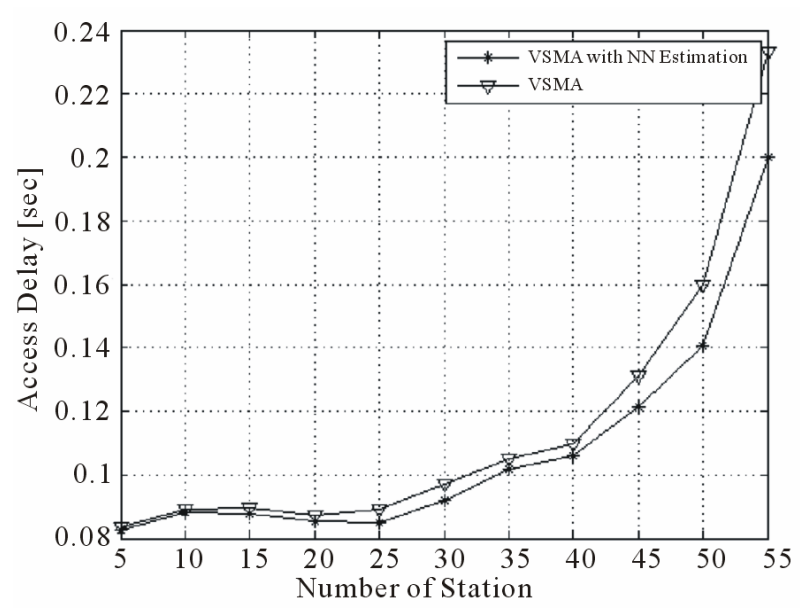

Figure 11. Comparison of access delay with and without neural network channel estimation.

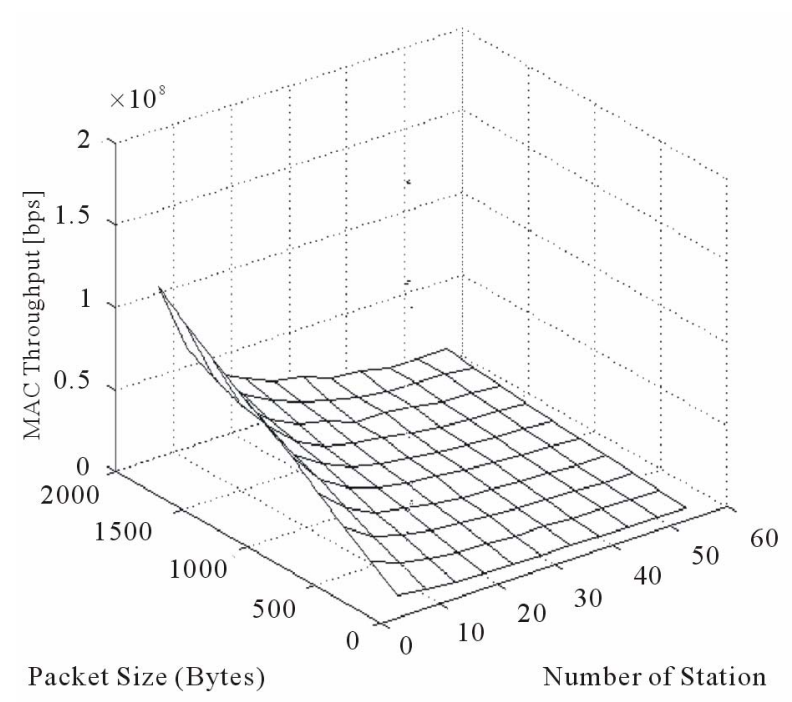

Figure 12. Throughput in VSMA (CSMA/CA + TDMA) with dynamic $\mathrm{CW}$.

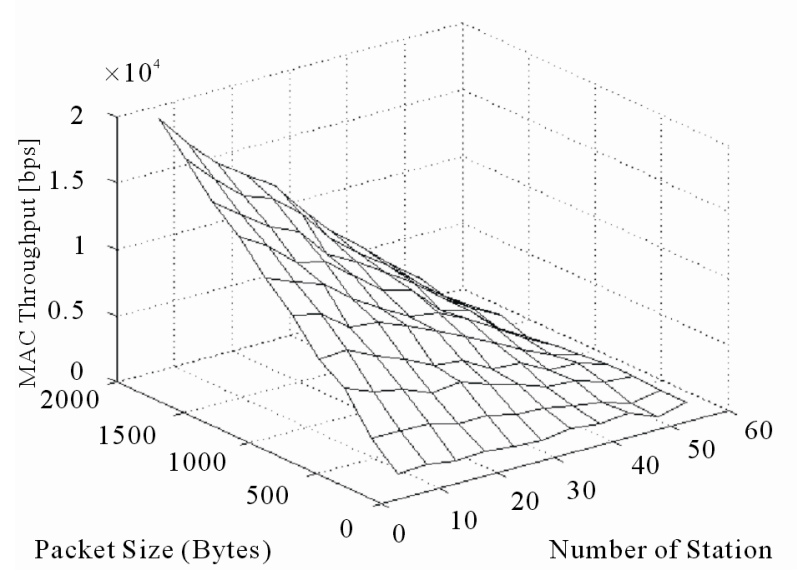

Figure 13. Excess throughput when channel estimation is done in VSMA algorithm. which has proper performance in mentioned condition, just in physical layer. VSMA techniques in MAC layer decreases access delay and increase throughput when neural network estimation is done. This is because of decreasing bit error rate in PHY layer. In addition to priority slot, dynamic CW is used in this paper to achieve better real-time communication. Analysis of MAC sublayer shows that frame size and number of stations are two parameters that directly influence throughput, but only the number of stations influence access delay. Impulsive noise and AWGN noise are used for simulation. In order to get better precision other noise must be used.

\section{Acknowledgements}

Thanks to Iran Telecommunication Research Center that undertook the moral and financial support of this research.

\section{References}

[1] A. Jeremic, T. A. Thomas and A. Nehorai, "OFDM Channel Estimation in the Presence of Interference," IEEE Transactions Signal Processing, Vol. 52, No. 12, 2004, pp. 3429-3439.

[2] Y.-H. Kim, S.-C. Kim and H. Myong Oh, “OFDM Receiver Performance Analysis with Measured Power Line Channel Model for Coded OFDM System, Power Line Communications and Its Applications,” 2005 International Symposium on Digital Object Identifier, Vancouver, 23-26 May 2005, pp. 201-205. doi:0.1109/ISPLC.2005.1430496

[3] Y.-H. Kim, S.-C. Kim and H. Myong Oh, “The Brazilian Power System and the Challenge of the Amazon Transmission," International Symposium on Power Line Communications and Its Applications, 6-8 April 2005, pp. 380-385. doi:10.1109/ISPLC.2005.1430496

[4] M. Pukkila, "Channel Estimation Modeling, Postgraduate Course in Radio Communications,” 2000. http://www.comlab.hut.fi/opetus/260/chan_est.pdf

[5] Alqueres and J. C. Praca, "An Application of the Singular Value Decomposition to OFDM Channel, Estimation,” Proceeding of 1991 IEEE Power Engineering Society Transmission and Distribution Conference, Dallas, 22-27 September 1991, pp. 315-320. doi:10.1109/TDC.1991.169475

[6] T. Necmi and S. M. Nuri, "Back Propagation Neural Network Approach for Channel Estimation in OFDM System, Wireless Communications, Networking and Information Security (WCNIS)," 2010 IEEE International Conference, Anchorage, 3-8 May 2010, pp. 265-268.

[7] L. L. Scharf, "Statistical Signal Processing: Detection, Estimation, and Time Series Analysis," University of Colorado Boulder, Boulder, 1991, pp. 325-327.

[8] X. Carcelle, "Power Line Communications in Practice", Artech House, Boston, 2009, pp.19-20. 
[9] H. Meng, Y. L. Guan and S. Chen, "Modeling and Analysis of Noise Effects on Broadband Power-Line Communications,” IEEE Transactions on Power Delivery, Vol. 20, No. 2, 2005, pp. 630-637. doi:10.1109/TPWRD.2005.844349

[10] R. Nakkeeran and A. Rajesh, "Performance Analysis of Powerline Communication for Local Area Networking," International Conference on Control, Automation, Com- munication and Energy Conservation, Tamilnadu, 4-6 June 2009, pp. 1-5.

[11] A. Rajesh, M. Rathinasabapathy and R. Nakkeeran, "Performance Analysis of Hybrid Protocol Based on Dynamic Contention Window for Power Line Communication Systems," First Asian Himalayas International Conference on Internet, Kathmandu, 3 November 2009, pp. 1-7. doi:10.1109/AHICI.2009.5340267 